\title{
LOCATION INFORMATION MANAGEMENT OF RFID-EQUIPPED BUILDING COMPONENTS
}

\author{
$\underline{\text { Ali Motamedi }}{ }^{1}$ and Amin Hammad ${ }^{2} *$ \\ ${ }^{1}$ Special Individualized Program, Concordia University, Montreal, Canada \\ ${ }^{2}$ Concordia Institute for Information Systems Engineering, Concordia University, Montreal, Canada \\ *Corresponding author (hammad@ciise.concordia.ca)
}

\begin{abstract}
Availability of accurate location information of components decreases the search time and increases the chance of using shared resources. In our previously proposed framework, RFID tags are attached to components at an early stage of the lifecycle and their memory is used for various purposes throughout the lifecycle. In this paper, we propose to store various types of location-related information (e.g., current location, temporary location) on RFID tags. Details about how these data can help users (e.g., inspectors) find components in the operation phase are further elaborated. Furthermore, the paper discusses how the location data on tags can provide the users with their current location and predefined paths in the building. For example, a user equipped with an RFID reader is able to see his/her approximate location on a floor plan based on the information read from surrounding tags without having access to any Real-Time Location System. A case study is designed and implemented in a high-rise university building.
\end{abstract}

Keywords: RFID, Location Information Management, Location-Based Services, Lifecycle Management

\section{INTRODUCTION}

Location data is essential for many processes related to supply chain management (SCM), facilities management (FM) and product lifecycle management (PLM). It enables employees to optimize their decisions with regard to the route they follow when looking for an asset, which results in significant improvement of resource utilization. Location tracking systems provide the potential for achieving savings depending on the selected technology [1]. Real-time Location Systems (RTLS) operate in both indoor and outdoor environments and have different characteristics, such as accuracy, precision, the need for fixed infrastructure, range and cost [2]. However, adapting RTLS technologies in complex buildings is complicated and costly.

In our previous research, a framework is proposed where Radio Frequency Identification (RFID) tags are attached to components at an early stage of their lifecycle and the memory on tags is used to store various types of data. Storing lifecycle information on RFID tags provides realtime data access that facilitates several processes during the lifecycle of buildings' components [3]. In this paper, we propose to store additional various location-related information (e.g., current location, temporary location, predefined routes) on tags' memory to be used by stakeholders throughout the lifecycle of the components. For example, the FM personnel can use these data for locating components. Our proposed approach is based on the assumption that relatively long-range RFID tags are attached to building components. These tags are not solely used for locating purpose, and their memory is used during the lifecycle for various applications. Additionally, location data on tags can assist building users (e.g., students of a university building) with estimating their current location in the building. For example, a user equipped with an RFID reader is able to see his/her approximate location on a floor plan based on the information read from surrounding tags without having access to any RTLS.

The objectives of the paper are: (1) to investigate the potential lifecycle location data to be stored on RFID tags, (2) to elaborate on methods to add and update location data on RFID tags, (3) to identify suitable target component 
types for location tracking using RFID data during the operation phase, and (4) to investigate the benefits of storing location information for locating assets and users.

\section{LITERTURE REVIEW}

\subsection{RTLS technologies}

The need for indoor positioning has been rapidly expanding in many fields in recent years [4]. Liu et al. [2] have reviewed the current wireless location sensing systems for indoor applications. Different performance criteria are discussed with several tradeoffs among them. Fuchs et al. [5] have reviewed indoor tracking methods focusing on the requirements of mission critical applications.

RFID can be used for indoor location sensing. Three major methods are used to locate RFID tags or readers: (1) triangulation, (2) proximity, and (3) scene analysis (fingerprinting) [6,7]. The first two methods require reference points with known positions to perform trilateration. The third method uses preexisting signal strength database (fingerprint map) together with Received Signal Strength (RSS) value. Radio Frequency (RF) interference among RFID components and some materials are the main limiting factors [6]. Li et al. [7] have reviewed several RFID-based indoor location sensing solutions and concluded that no single solution meets all the criteria for success, and the adaptability of these solutions is uncertain.

\subsection{RFID-assisted lifecycle information management}

The use of attached RFID tags for lifecycle management has been proposed in the aerospace industry for storing unique ID and important lifecycle information on tags attached to aircraft parts for enhancing inspection and repair processes [8]. Several studies suggested storing data related to components on attached RFID tags [3,9,10]. Only few studies explored on the potentials of storing location-related data on RFID tags (e.g., [11]).

The framework developed in our previous research proposed adding structured information taken from the Building Information Model (BIM) database to RFID tags attached to the building components [3]. Having data related to the components readily available on the tags provides easy access for stakeholders that need to access the data regardless of having real-time connection to a central database. In this framework, every component is a potential target for tagging. Having standard tags attached to components results in a massive tag cloud in the building. The target components are tagged during or just after manufacturing and are scanned at several points in time during the lifecycle. The scan events are for reading the stored data or modifying the data based on the system requirements and the stage at which the scan is happening. The scanned data are transferred to different software applications and processed to manage the activities related to the components (e.g., inspection and maintenance activities).

Considering the limited memory of the tags, the subset of BIM data stored on these tags has to be chosen based on the requirements of the tasks. The data on a tag are changing during the lifecycle and different software applications use and modify the data with different designated access levels [10].

\section{PROPOSED APPROACH}

\subsection{RFID memory for storing lifecycle location data}

In our framework, components are scanned and the data on the tags are updated before each stage of the lifecycle. Consequently, the location-related data can be stored/updated during the same data update event. The proposed location information that can be stored on RFID tags are:

A: Final location is defined at the design phase for fixed components.

B: Subcomponents' locations are the information about the parts inside the component, e.g., mechanical and electronic parts such as controllers and power units. These data can be used in the assembly line to help operations such as welding and part installation. These data are also useful to help detaching the faulty part for repair purposes or could be used to dismantle the component at the end-of-life.

C: Attached parts' locations are the data about ports and connection points that connect the component to its adjacent units (e.g., HVAC ducts). These data are most useful at the assembling and installation stages and can 
also be used at the operation phase where the component is detached for repair or maintenance purposes.

D: Temporary location is any location that the component may be stored at other than its final location. The components are stored in various locations (e.g., storage, yard, shelf, floor, and warehouse) during their supply chain and the temporary storage location can be recorded to help moving the components.

E: Delivery lot information can be used at shipping and transportation stages and managed by supply chain management software. The data can also be used for inspection and quality control purposes.

F: Destination site is used at transportation and receiving stages where the components are transported and delivered to designated locations.

G: Disposal location is used to ensure that the component is disposed at the right location

$\mathrm{H}$ : Current location information recorded on long-range RFID tags can be used to locate components. This field of memory will be further discussed in Subsection 3.2.

I and J: Predefined routes, floor plans/maps can be used for navigational purposes. The FM personnel, emergency responders or general facility users can use this data to download the floor plan and navigational information.

$\mathrm{K}$ : Locations of other components include the relative locations of other components that do not have tags attached to them or have tags with short readability range.

L: Previous locations are the history of important previous locations of a component.

The above-mentioned information can facilitate operations, such as locating components, warehouse management, shipping and transportation, assembling and installation, supply chain visibility, site management, quality control, dismantling, repair and navigation. For example, at the design phase, the ID and the final location of the component are created and communicated with the manufacturer in the ordering process. While the final location information is written on the tag at an early stage in the lifecycle, a variety of temporary location information is stored and used at various stages. Thus, in order to use relevant location information, series of read/write events have to be executed during the lifecycle. Fig. 1 shows some of the possible location-related data that can be stored on a tag attached to a generic component. The required location information is determined based on the type of component and the processes involved in its lifecycle. The figure also shows the lifecycle stages where the location data can be used. The white, shallow-grey and dark-grey colors show whether the data is updated, read or written, respectively, on the tags' memory at that specific lifecycle stage.

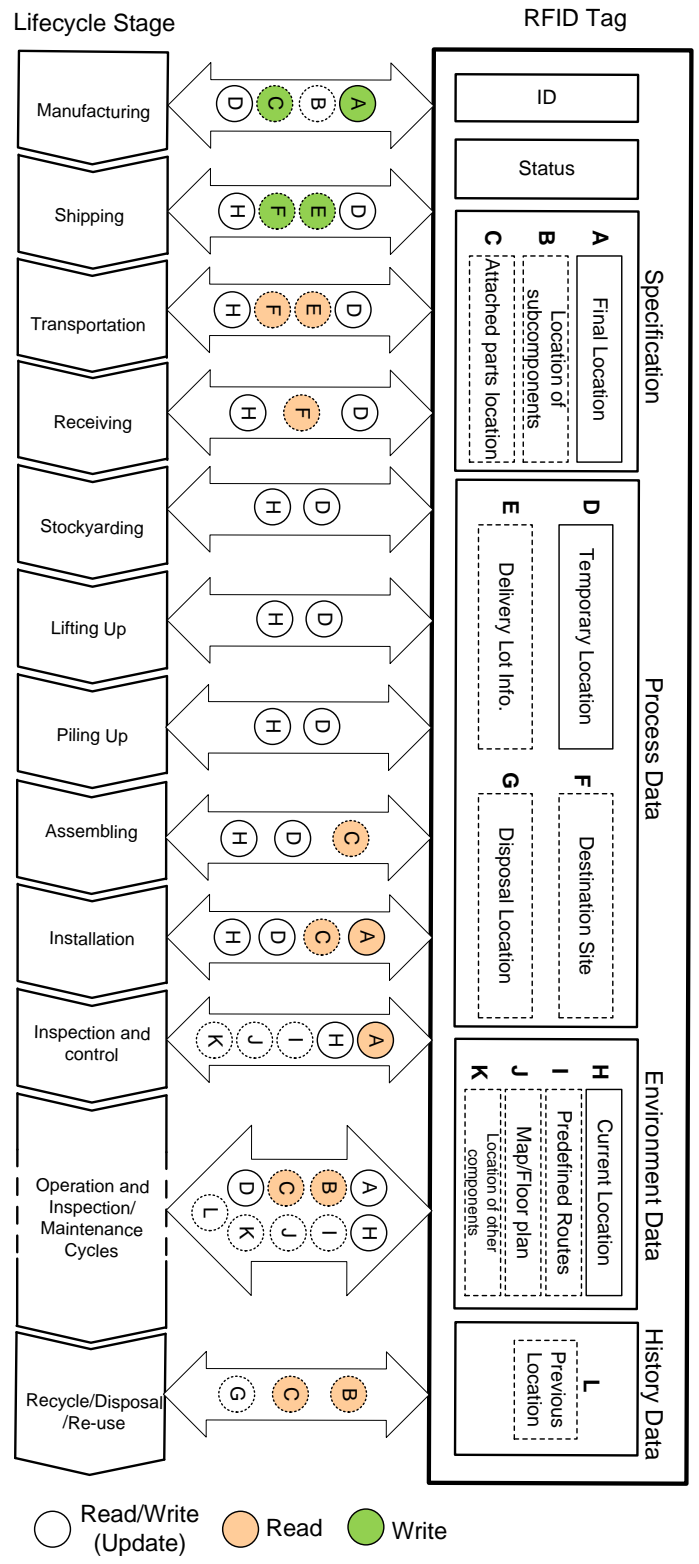

Fig. 1 Examples of Lifecycle Location-Related Data 


\subsection{Current location data}

Finding components in a building for tasks such as preventive maintenance and repair is time consuming. Hence, accurate and updated location data are invaluable for facilities managers and operators.

There are two methods for providing the current location of a component: (1) The current location is detected by RTLS technologies, (2) The current location data are stored on long-range RFID tags attached to the component to help locating the component for a user who is equipped with an RFID reader. In the first method, users need to have access to the RTLS interface (e.g., web service, middleware) to query and retrieve the location of a specific component (Fig. 2(a)). In the second method, location data are available on tags and the user can read the data and locate the component even if it is hidden, obstructed or stored in closed areas, without having access to any RTLS (Fig. 2(b)).

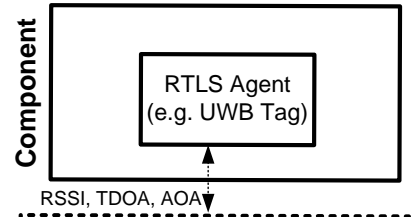

RTLS Services (e.g. UWB middleware) RTLS Services (e.g. UWB middleware),

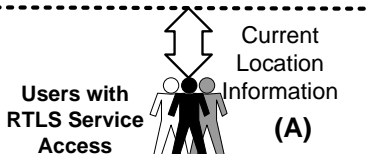

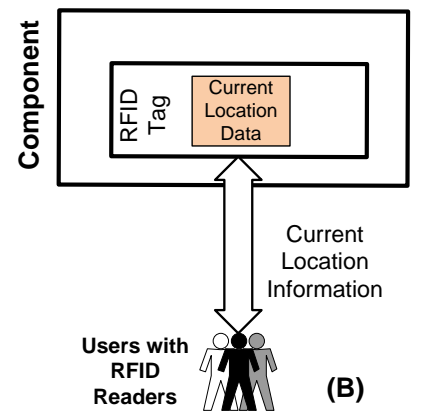

(B)
Fig. 2 Location Tracking Methods

By storing the updated current location data on the attached tags, the user can read the RFID data from a distance and retrieve the location information. In this scenario, the user needs to have a preloaded floor plan to be able to find components. By storing additional navigation data (e.g., maps and floor plans) the user can find the component in the building without having any preloaded floor plans.

\subsubsection{Categorization of components at operation phase}

Building components can be categorized in four major groups: (1) Fixed components (e.g., boiler, door, HVAC ducts) that do not move after installation. However, they may be moved during the lifecycle for repair purposes; but they should be installed back in their original location; (2) Semi-fixed components that are moved infrequently. The movement can be planned, where a component is moved by FM personnel and the movement is recorded (e.g., fire extinguishers, furniture) or, unplanned where a component is moved per use by users based on special needs (e.g., computers or lab equipments); (3) Movable components (e.g., shared ladder, cart, containers) that are frequently moved. Moving these components does not require special permission from the FM department; and (4) Temporary components that are not owned by building owners and are used for a limited period of time (e.g., scaffolding, tools of a contractor).

\subsubsection{Suitable location tracking platform}

Finding the location of components is desirable during the operation phase regardless of their category. RTLS technologies are capable of providing real-time location information for assets that are equipped with RTLS agents (tags). However, providing RTLS infrastructure inside the building is costly and imposes tremendous amount of technical design and implementation issues such as the scalability of RTLS.

Considering that a large portion of available assets in the building are fixed or semi-fixed, accurate tracking of their location in real time is unnecessary. FM software contains location information of fixed and semi-fixed components on floor plans or in asset databases. Consequently, in order to locate these components, FM databases can be referred to instead of data provided by an RTLS service. In contrast with fixed and semi-fixed components, RTLS systems are more suitable for tracking the location of movable components.

This paper proposes using current location data on RFID tags for fixed and semi-fixed components that follow planned movement patterns. However, because the data is manually updated and the location update is not in realtime, this method is not suitable for movable components. Since the current location data is available locally on tags and can be read from distance by handheld readers, this method does not require any infrastructure. Although this method is only suitable for fixed and semi-fixed components, these components are the main target of 
maintenance activities in the building. This method can also be used for some temporary components that are not movable in the building. These components can be equipped with RFID tags and their locations can be updated on the tags by FM personnel.

\subsection{Location tags}

Attaching long-range tags with large memory capacity to all fixed and semi-fixed components may not become financially feasible in the near future. In order to benefit from the proposed location tracking method using RFID data, specific long-range tags can be attached to selected components and assigned to a space to store location information related to a set of components in that space. In this scenario, other components can be equipped with short-range passive tags. Consequently, when a user tries to locate a component, the data on the location tag is read from a distance which contains location data for all components in that space including the target component. These location tags can also be equipped with large memory chipsets that contain floor plans and navigational information. The location information is updated on location tags when a fixed component is installed in that specific area, or when a semi-fixed component is moved to/from that specific area by FM personnel.

\subsection{Using location information to position the user}

In addition to locating assets, current location data on tags can help users finding their estimated locations in the building. This method can be used in complex facilities, such as hospitals or universities. There are two major scenarios for the user to estimate his/her location from surrounding tags: (1) the user scans a visible tag on a component or a location tag that is located in a close proximity and read the current location data of the tag. Consequently, the user can be provided with his/her current location information, (2) the user scans the environment and reads the location data of surrounding tags to be used for RFID location estimation techniques.

As explained in Subsection 2.1, proximity method is used to locate a moving reader by measuring its distance from tags with known locations. In our proposed framework, tags attached to fixed component store their precise location information; consequently they can act as reference points for RFID indoor location sensing technologies. RFID tags can also store signal strength database for surrounding area. The user can retrieve the signal strength fingerprint map from detected tags and estimate the location of his/her reader. In this method, tags are not necessarily visible or in a close proximity.

\section{CASE STUDY}

Active RFID tags with relatively long-range $(100 \mathrm{~m})$ and $32 \mathrm{~KB}$ of storage together with handheld readers are employed. Memory of tags contains information about the components as well as location specific data. The information is saved in Extensible Markup Language (XML) format where XML-tags are partially taken from definitions in IfcXML [12] to facilitate the mapping of BIM data with the data on tags.

RFID tags are attached to fire extinguisher cabinets that are inspected frequently and are highly spread in the building. In the first scenario, location data is used to help FM personnel finding extinguishers. FM personnel use the handheld software to scan the environment and the software shows detected tags on the floor plan. Detected components are shown on the floor plan with dots with different colors that specify the type of the component (Fig. 3(a)). FM personnel can select the desired tag from the floor plan and retrieve detailed information (Fig.3(b)). In the second scenario, RFID data are used to help users detect their location. A facility user (e.g., a student) scans the environment and the software shows the floor plan of the building with dots representing detected tags. The size of dots is proportional to RSS value for each detected tag. The user will be able to find his/her location visually from the floor plan. Moreover, the user is able to read public data that is stored on the tag to get more information about the environment (e.g., department's name, emergency exits). In order to improve the accuracy of the system, the power of the reader is reduced to detect only tags located in a close proximity. RFID tags used in this case study are equipped with Light-Emitting Diodes (LED) that help the user to visually locate the closest tag.

\section{CONCLUSIONS AND FUTURE WORK}

The current paper is an extension to the previous research of the authors by introducing additional location-related 
information to be stored on RFID tags. The paper discussed the usage of current location data to assist FM personnel with finding some asset types and to help users estimating their location in the building. Moreover, the idea of having location tags is discussed. The case study showed that location data saved on tags attached to fixed components can help FM personnel locate components more efficiently.

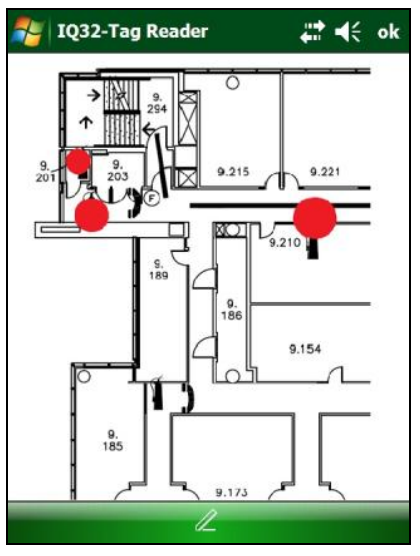

Fig.3 (a) Tag location

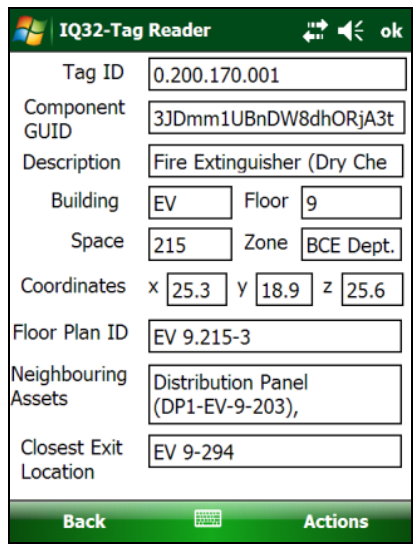

(b) Tag data
Although, the research introduced some lifecycle locationrelated information to be stored on tags, other types of location data that can be used during the lifecycle, especially throughout the supply chain (e.g., storage shelf), can be defined and added to the framework. Moreover, details about providing users with navigational aid including floor plans and routes extracted from a BIM can be further investigated.

Although the case study showed the technical feasibility of the proposed approach, several challenges should be addressed to make the vision practical and financially feasible. Some of these challenges related to active RFID tags are: (1) lack of international standards, (2) limited memory and readability range, (2) limited battery life, and (3) high cost.

\section{ACKNOWLEDGEMENT}

The authors would like to acknowledge the contributions of Mr. Kehinde Adetiloye in realizing the case study. Execution of the case study could not be done without the help provided by Concordia University Facilities Management Office and Identec Solution.

\section{REFERENCES}

[1] Kelepouris, T. and McFarlane, D., "Determining the value of asset location information systems in a manufacturing environment", Production Economics, Vol.126( 2), pp. 324-334, 2010.

[2] Liu, H. and Darabi, H., "Survey of wireless indoor positioning techniques and systems", IEEE Transactions on Systems, Man and Cybernetics, Vol.37(6), 2007.

[3] Motamedi, A. and Hammad, A., "Lifecycle Management of Facilities Components Using Radio Frequency Identification and Building Information Model", IT in Construction, Vol.(14), pp. 238-262, 2009.

[4] Khoury, H. and Kamat, V., "Evaluation of position tracking technologies for user localization in indoor construction environments", Automation in Construction, Vol.18(4), pp. 444-457, 2009.

[5] Fuchs, C., Aschenbruck, N., Martini P. and Wieneke M., "Indoor tracking for mission critical scenarios: A survey", Pervasive and Mobile Computing, Vol.7(1), pp.1-15, 2011.

[6] Papapostolou, A. and Chaouchi, H., "RFID-assisted indoor localization and the impact of interference on its performance", Network and Computer Applications, Vol.34(3), pp. 902-913, 2011.

[7] Li, N. and Becerik-Gerber, B., "Performance-based evaluation of RFID-based indoor location sensing solutions for the built environment", Advanced Engineering Informatics, in Press, Available online 23 March, 2011.

[8] Harrison, M.. "Guidelines for lifecycle ID \& data management", AEROID-CAM-014, 2007.

[9] Ergen, E., Akinci, B. and Sacks, R.. "Life-cycle data management of engineered-to-order components using RFID, Advanced Engineering Informatics, Vol.21(4), pp. 356-366, 2007.

[10] Motamedi, A., Saini, R., Hammad, A., Zhu, B., "RoleBased Access to Facilities Lifecycle Information on RFID Tags", Advanced Engineering Informatics, in Press, 2011.

[11] Yeates, A., Zhu, Y., Belvin, T. and O'meara, B., "Wireless self-describing buildings", US Patent 6963282, 2005.

[12] Nisbet, N., and Liebich, T., "ifcXML Implementation Guide", Modeling Support Group, International Alliance for Interoperability, 2005. 